\title{
Evolutionary developmental robotics: Improving morphology and control of physical robots
}

\author{
Vuk Vujovic ${ }^{1}$, Andre Rosendo ${ }^{2}$, Luzius Brodbeck ${ }^{1}$, and Fumiya \\ Iida $^{2}$ \\ ${ }^{1}$ Institute of Robotics and Intelligent Systems, ETH Zurich, Zurich, Switzerland \\ ${ }^{2}$ Department of Engineering, The University of Cambridge, Cambridge UK \\ E-mail: andre.rosendo@eng.cam.ac.uk
}

\begin{abstract}
Evolutionary algorithms have previously been applied to the design of morphology and control of robots. The design space for such tasks can be very complex, which can prevent evolution from efficiently discovering fit solutions. In this article we introduce an evolutionary-developmental (Evo-devo) experiment with real-world robots. It allows robots to 'grow' their leg size to simulate ontogenetic morphological changes, and this is the first time that such an experiment has been performed in the physical world. To test diverse robot morphologies, robot legs of variable shapes were generated during the evolutionary process and autonomously built using additive fabrication. We present two cases with Evo-devo experiments and one with Evolution, and we hypothesize that the addition of a developmental stage can be used within robotics to improve performance. Moreover, our results show that a non-linear system-environment interaction exists, which explains the non-trivial locomotion patterns observed. In the future, robots will be present in our daily lives, and this work introduces for the first time physical robots that evolve and grow while interacting with the environment.
\end{abstract}

Keywords: Evolutionary robotics, Developmental robotics, Evo-devo, Genetic algorithms, Ontogeny, Phylogenetic. 


\section{Introduction}

The design automation of artificial machines -physical or virtual- remains an interesting research challenge. In the early nineties, Sims presented his work on the simulated evolution of virtual life forms [14]. Further work explored the application of evolutionary algorithms in many artificial scenarios $[25,8]$. The goal was to exploit the ability of evolutionary processes to optimize such complex design problems, i.e. to improve the robot's performance by implementing a task-specific fitness function. This way, both the control $[20,10]$ and the morphology [11] of the robot can be subject to the design automation, and often both aspects evolve in parallel $[12,15]$.

When evolving real-world robotics the challenge for evolutionary algorithms is to cope with noise and disturbance from the physical world. Given the available computational power, most systems try to circumvent this problem with simulated environments, some of which are eventually transferred to the real-world $[6,4,1]$. While Hornby et al.[4] propose a computer simulated antenna that outperforms humandesigned antennas in the real life, Mouret et al. [1] present a series of motions that confirm the physiological representation of a robot and the compatibility with its internal virtual model. However, the transfer of simulated results into a real system is affected by a phenomenon called Reality Gap [19]. A priori, a simulation can never exactly model the behavior of a physical robot in its environment due to stochastic disturbances and over-simplification of ground friction. Thus, a solution that performs well in simulation is not necessarily good when implemented on a physical system. So as to overcome this limitation, several approaches have been presented: Koos et al. [26] co-optimized the transferability of evolved solutions while Floreano et al. evolved a homing navigator by lifting design constraints, which define the interaction with the environment [3]. Similarly, several other works have demonstrated the evolution of robot controllers in the real-world $[20,21]$ and how robots can even learn to update their internal morphological representation through interactions with the environment [7]. In our previous publication we have shown that the evolution of robot morphologies in the real-world is also a possibility through an automated assembly system [15].

The search space that the automated evolution has to cover can be very large, sometimes even considered infinite. Given the limited speed at which robots can be evaluated in the real-world, this can prove prohibitive. Biology, however, shows that plants and animals adapt their body structures to the environment, not only over generations (phylogenetic) but also during their lifetime (ontogenetic) [2]. For example, plants adjust their leaf biomass to the lighting conditions [28], and frogs have been reported to be capable of repeatedly changing their skin texture [13]. The field of developmental robotics aims to build robots that, throughout their lifetime, adapt to their environment [18]; and both morphology and control are potential targets for development [30]. Even though development is usually approached by biologists $[27,5]$, computer scientists can also devise simulations to study ontogenetic changes in virtual organisms $[8,22]$. As it has been shown that simulated morphological changes 
can improve the evolution of virtual robots [9] and animats [17], we hypothesize that such improvement can, in the form of a developmental stage, be combined with an evolutionary stage and improve the fitness of real-world robots.

In this work we demonstrate a combination of evolutionary and developmental processes for the automated design and generation of physical robots, as illustrated in Fig. 1. On the evolutionary timescale, a population of robots is evolved, as the best solutions from each generation are selected and mutations and crossovers are used to perpetuate their genotypes. During its lifetime, each robot assumes three developmental stages, and at each stage some of its parts (e.g. the leg length) grow based on the robot's real-world interaction. We present experimental results that imply that the introduction of a developmental process potentially improves the evolutionary process when an appropriately chosen growth function is selected. Evolutionary processes improve robots in a phylogenetic approach, and this paper studies for the first time a comparison between different developmental parameters for physical robots capable of changing their morphology over time.

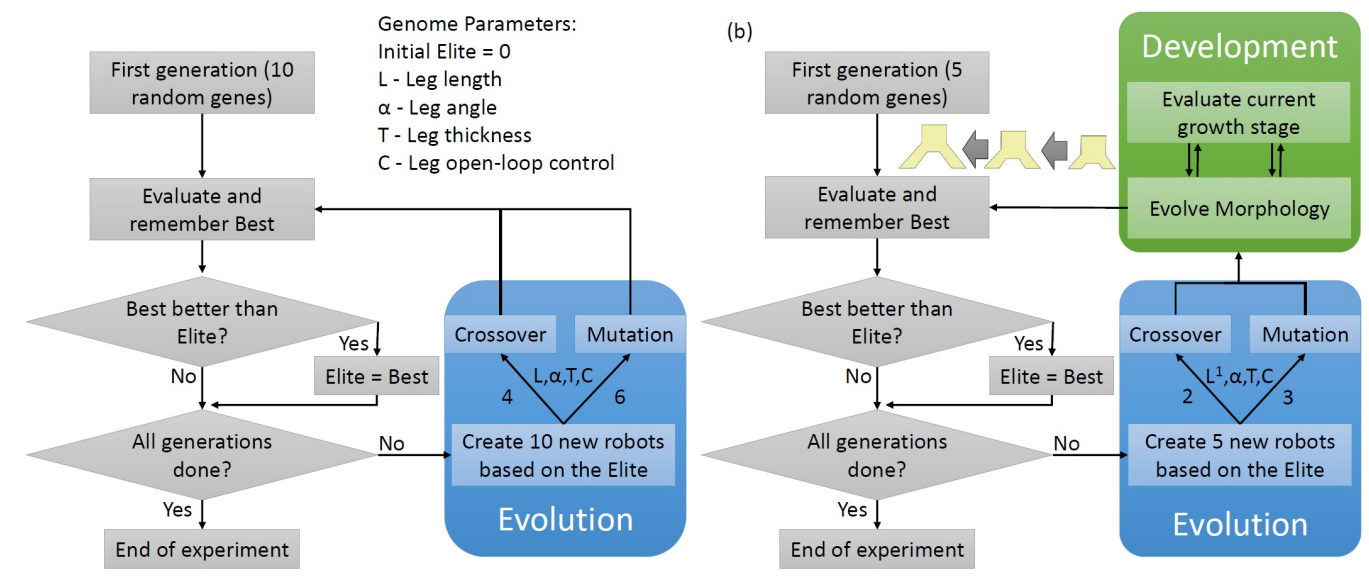

Figure 1. This figure shows (a) the evolutionary process and (b) the nested evolutionary and developmental process for robot morphologies. Both processes take place at different timescales, although the difference is not comparable to the biological processes. The implementation of the growth process is decisive for the success of our approach.

In this paper we apply Evo-devo in order to develop robots able to walk over flat horizontal space, the fittest robots being those capable of travelling the longest distance within ten seconds. An external robotic arm is employed to implement our Evo-devo process on a physical system, as it is capable of autonomously fabricating and testing evolved legged robots. Contrasting with our previous work [15], we increase the variety of shapes that such legged robots can assume by additively fabricating soft legs. Such legs follow gene design parameters, they are built by the robotic arm on-the-fly with hot glue, and are finally combined with other components to form the legged robots. 


\section{Materials and methods}

\subsection{Evolution of physical robots}

Evolutionary algorithms are optimization methods inspired by the evolutionary mechanism found in nature and defined by Charles Darwin. While using genomes to define individual robots, these evolutionary algorithms test behaviors and store the best performances to further create new robots from combinations of the best ones.

The implementation of the evolutionary algorithm here consists of an evolutionary parameter search, with an initial population of legged robots which evolves from one generation to the next. In each generation, an internal search iterates over the whole set of individuals of the current generation to physically build new robots based on their encoded genotypes and experimentally evaluate their fitnesses. The robots with higher fitness values are selected as the elite and are used to generate the next generation of robots through crossover and mutation. The idea behind crossovers and mutations is to balance the exploration of the design space and the exploitation of strong features of the already tested robots. The mutation can randomly change each parameter in a robot's genotype with probability 0.7 , while the crossover function combines the two parent genomes through a randomly decided one-point crossover.

Unlike other authors, here the terms "robots" and "robotics" are, in a strict sense, used to refer to real-world agents, as opposed to virtual agents. Moreover, the phenotype (i.e. the observable traits) is the physical representation in the legged robot, while the encoding of this potential solution defines the genotype. The chosen fitness function is the distance traveled during ten seconds of locomotion. Together with the robot's morphology, the control is co-evolved during our evolutionary optimization. All parameters considered during the evolution of the legged robot are detailed in Fig. 2.

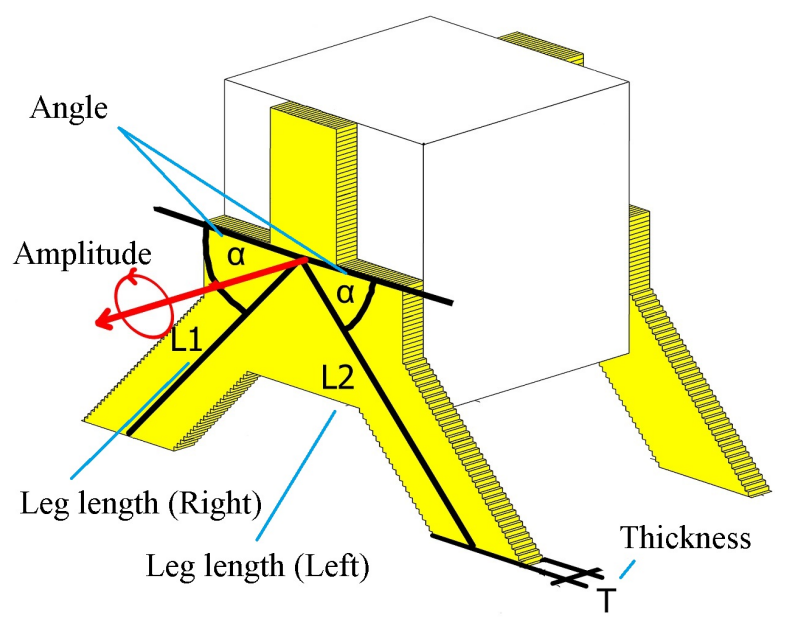

Figure 2. Pictogram with five different parameters used to evolve and develop the legged robots. $L_{1}$ and $L_{2}$ represent left and right legs, respectively. $T$ is the leg thickness in layers, $\alpha$ is the leg angle in the horizontal plane, and Amplitude is the amplitude of the control parameter for the rotation of legs during locomotion. 


\subsection{Evolution and Development of Robots}

Aiming to improve the evolutionary process and to reduce the complexity of the fitness landscape, a developmental part is added to our second experiments to achieve an Evodevo process. The evolutionary part, which has been previously defined, is extended by a nested developmental search - a unidirectional search (only growth is allowed) of specified parameters. Within robotics the novelty of this search is that the robots' morphology changes in multiple stages through a 'growth function', and the aim is to understand how ontogenetic morphological changes can affect robotics.

Figure 1(b) shows the flowchart of such a system, where the developmental stage is executed for each individual of a population. The growth function determines how leg length changes from one developmental stage to the next. In this article, two different growth functions are explored and applied to two shape parameters of the legged robots.

The first parameter that can be altered in the developmental process is the leg length $L$. The initial leg lengths are defined by the genes $L_{1}^{1}$ and $L_{2}^{1}$ within its genome. For Evo-devo processes, specifically, the maximum value for $L_{1}^{1}$ and $L_{2}^{1}$ is half of the maximum $L$ value. From stage $n$ to $n+1$ both legs are increased by $\Delta L$ depending on the robot's fitness $f^{n}$ as shown in (2). This function is designed such that a larger physical growth is given to robots that do not travel long distances (low fitnesses grow more), and we aim to simulate a self-stabilizing mechanism, as good behaviors remain almost unchanged and bad performing robots are drastically altered. From an energy perspective, however, lower fitnesses intuitively represent little to no movement, and the energy saved with the lack of locomotion can be used to boost the ontogenetic morphological change. The parameter $b$ is the minimum growth of the leg, $A$ is the amplitude of growth and $k$ is the coefficient of exponential decrease of growth with respect to fitness. The actual parameter values for the Evo-devo experiments are listed in table 1.

$$
\begin{aligned}
& L^{n+1}=L^{n}+\Delta L \\
& \Delta L=A \exp (k f)+b
\end{aligned}
$$

The minimum leg thickness adopted is manufactured with 2 layers of additive material, and each layer is approximately $1 \mathrm{~mm}$ thick. While Evolution experiments encode 2, 3 and 4 layers as design possibilities, the developmental approach starts the first developmental stage with 2 layers and adopts fixed increments of $1 \mathrm{~mm}$. The limitation of the number of layers during construction restricts the encoding of the thickness gene, and this prevents the system from developing length and thickness in similar ways.

$$
\begin{aligned}
& T^{1}=2 m m \\
& T^{n+1}=T^{n}+\Delta T \\
& \Delta T=1 m m .
\end{aligned}
$$


The second parameter is the leg thickness $T$. The minimum thickness of $2 \mathrm{~mm}$ was chosen, as preliminary experiments hinted that thinner limbs could not support the weight of the robot. Upon reaching its last developmental stage, the fully grown legged robot is evaluated and the best genes carried forward. The rationale behind this decision is to mimic a reproductive age; the best children are not necessarily the best adults, and another adult would seek the best partner based on current information, and not on its past performance.

Table 1. Evo-devo algorithm parameters within the developmental stage

\begin{tabular}{|c|c|c|}
\hline Parameter name & Value & Description \\
\hline Generations & 5 & Number of generations evaluated \\
\hline Development stages & 3 & $\begin{array}{l}\text { Number of developmental stages each indi- } \\
\text { vidual experiences }\end{array}$ \\
\hline Length growth & Eq. (2) & Function defining length increments \\
\hline Thickness growth & Eq. (5) & Function defining thickness increments \\
\hline A & $12 \mathrm{~mm}$ & $\begin{array}{l}\text { Amplitude of growth according to develop- } \\
\text { ment function }\end{array}$ \\
\hline $\mathrm{k}$ & -0.05 & $\begin{array}{l}\text { Exponential descent rate of development } \\
\text { function }\end{array}$ \\
\hline $\mathrm{b}$ & $3 \mathrm{~mm}$ & Minimum leg growth during development \\
\hline Length increments & $3 \mathrm{~mm}$ & Minimum $45 \mathrm{~mm}$ - Maximum $72 \mathrm{~mm}$ \\
\hline Amplitude increments & $5^{\circ}$ & Minimum $30^{\circ}-$ Maximum $50^{\circ}$ \\
\hline Angle increments & $10^{\circ}$ & Minimum $20^{\circ}-$ Maximum $60^{\circ}$ \\
\hline
\end{tabular}

\subsection{Materials}

The legged robots are automatically built by a robotic arm, which additively fabricates the robot legs and assembles these legs with a motor module. The system is capable of running for 5 hours without human interference, at which point an user is required to add new raw materials to allow the construction process to continue. The developmental growth, mentioned in the previous section, is reproduced by incrementally replacing the previously glued legs with new, longer ones.

2.3.1. Robotic assembly The robotic arm (Universal Robots UR5) has six degrees of freedom and is equipped with two main tools for this project, as shown in Fig. 3. It contains a hot melt adhesive extruder, controlled via the robot controller and capable of releasing a continuous stream of hot melt adhesive to unite cubes or fabricate parts. The nozzle diameter of $0.2 \mathrm{~mm}$ allows for a thin enough glue thread for the fabrication of precise parts. The material is released at $165^{\circ} \mathrm{C}$ and hardens within seconds. 


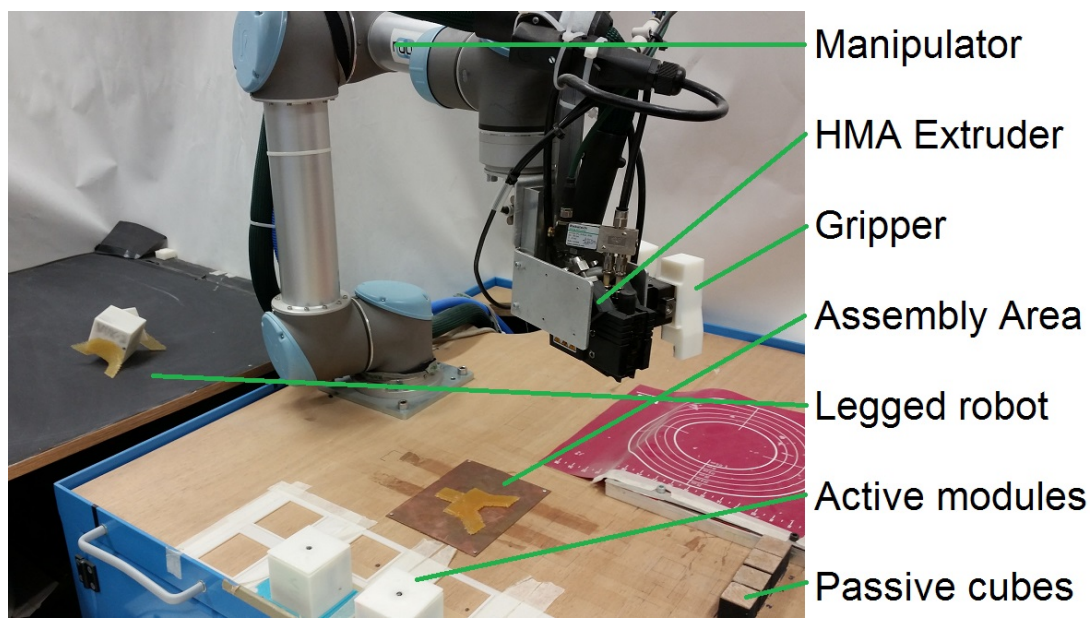

Figure 3. Hardware setup of the automatic fabrication system based on a UR5 robotic manipulator equipped with HMA supplier and gripper. The legged robots are assembled from additively fabricated parts, active modules and passive cubes. After evaluation, which is observed by a webcam, the manipulator removes the legged robot from the testbed and creates a new one.

A second tool is a pneumatic parallel gripper and it is mounted next to the nozzle. It is powered by a compressor and also controlled through a digital output of the robot controller. The gripper is used to pick differently sized objects and it is therefore designed with stepped fingers such that the lower part of the gripper can pick objects of roughly $6 \mathrm{~cm}$ width and the upper part can pick objects of $4 \mathrm{~cm}$ width.

Automated fabrication of leg shapes An important improvement in this study over our previous work [15] is the capability to generate glued parts in real time. The robot legs, as shown in Fig. 3, can assume different morphologies depending on the specific parameter values.

A fused deposition modeling process $[29,16]$ with hot melt adhesive material (ALFA Klebstoffe, ALFA H 5500/30) is employed to build the robot legs. A 3D voxel grid is generated from the encoded leg shape parameters, and this voxel is converted to an STL file to be processed by a slicer program. The output from the slicer is used to define the waypoints of the robot, which guides the nozzle to deposit material and finalize the fabrication process.

The hot melt adhesive is provided by a melting tank (Robatech, Concept B12). The hot melt material is used for additive fabrication of leg shapes and for the bonding assembly of individual elements of the legged robots. A piston pump provides a pressure of 4 bar which ensures a steady supply of hot melt adhesive.

2.3.2. Modules The legged robots are assembled as a combination of active modules, passive modules and additively fabricated parts. The design of the modules are as follows: 
Active modules The modules are cubic-shaped and their cases are made from acrylonitrile butadiene styrene (ABS). A servo motor and diverse electronic components are embedded in it, as an adaptation from previous modules [15] with improvements in both micro-controller software and modularity of the hardware. This ensured that modules are decoupled from controlling system and are able to achieve complex behavior and control. Also, the modularity of the hardware allowed for a faster battery change and to easily reprogram the modules.

Fig. 4 shows the internals of an active module. The module has a cubic shape with a side length of $60 \mathrm{~mm}$. It features a LiPo rechargeable battery that is easy to change as it is stored in a separate compartment of the module and is connected to the power switch via a single connector. The batteries can power the module for about $4 \mathrm{~h}$ to $6 \mathrm{~h}$ reliably. The module furthermore contains a micro-controller (Arduino, Mini Pro), which is running the control algorithm for an oscillatory movement of the servo motor (HS-82MG). This movement is defined by a PID control loop with an amplitude which is inversely proportional to its frequency. Also, the module features a Bluetooth RN-42 for communication with a Matlab enabled desktop PC which communicates with the module.

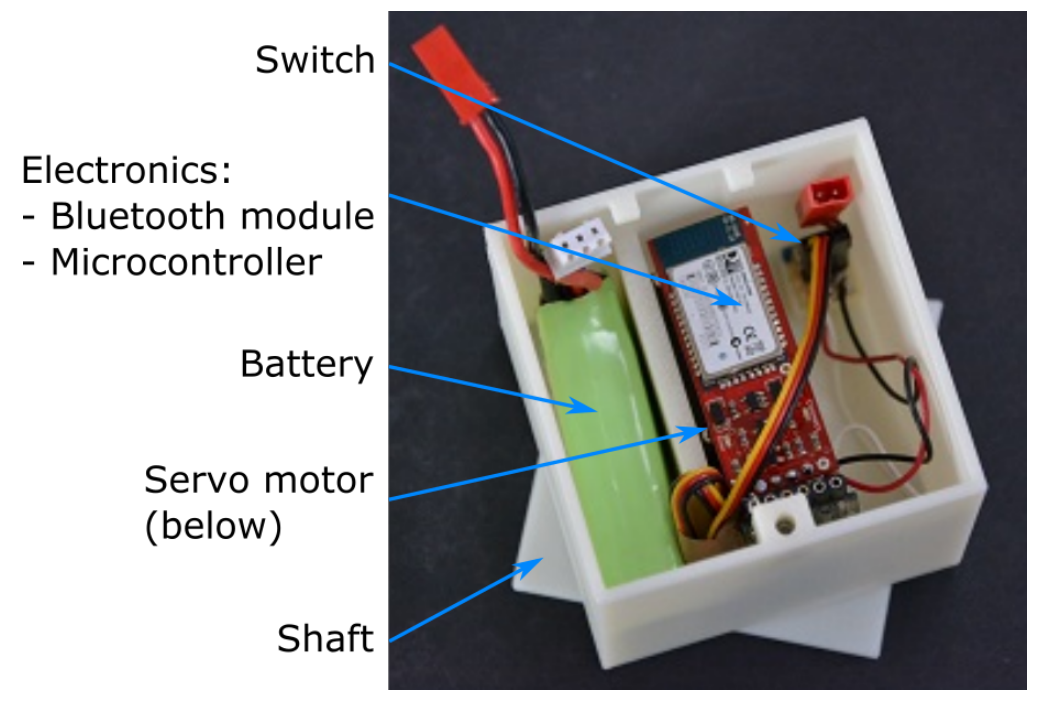

Figure 4. Internal components of an active module. The left side of the module contains a LiPo rechargeable battery, the center part houses electronics and a servo motor underneath, which is connected to the shaft. The right side includes the main switch and the power distribution.

Passive cubes These modules are simple wooden cubes used as handles to manipulate the additively fabricated parts. Their surface sticks to the glue from the fabricated parts and ensures that it remains flat during pickup.

2.3.3. Testbed setup This section describes the testbed and the additional elements of the hardware setup surrounding the testbed, which are a cleaning tool and the webcam 
system. The testbed itself is a $67 \mathrm{~cm} \times 63 \mathrm{~cm}$ wooden plate covered with black paper sheet to improve contrast with the white module for easier image analysis.

Cleaning tool The cleaning tool is a device that the robotic arm uses to remove legged robots from the testbed once the experiment has finished, hence ensuring that all experiments start with an empty testbed. The tool is designed to push the legged robot over the edge of the testbed (from where they drop into a padded box). The cleaning tool is stored on one side of the testbed on small holders with a magnetic system.

Webcam system The system of two Logitech webcam C930e cameras is used to record the experiment and evaluate the fitness of the legged robots. One camera is used to observe the distance traveled from the top. The fitness of the legged robots, i.e. the distance traveled during the evaluation phase, is automatically extracted from this camera's footage using image analysis. The second camera records the behavior to foster a better understanding of locomotion strategies and is thus placed at the side of the testbed.

\subsection{Experimental method}

In juxtaposition with computer simulations, real-world experiments require rawmaterials, maintenance of parts and, more importantly, time to assemble robots. While results from similar works in a simulation environment $[14,8,11]$ can reach thousands of individuals, the physical construction of our robots limited the total number of individuals. The authors considered the integration between physical and virtual experiments to expedite the process, but the reality gap associated with the highly deformable contact of glue with the floor rendered simulations useless.

Our experiments are separated in three different stages: the Evolution experiment, Evo-devo L and Evo-devo LT, as defined below:

- Evolution process without development. From each genotype, one physical robot is generated and automatically tested to evaluate its fitness at the locomotion task (see Fig. 1 (a)). This experiment consisted of five generations and the first generation was initialized with ten randomly generated robot genomes. All following generations carry over the saved genes of two elite agents of the preceding generations and use these to produce ten new randomized agents, with four through crossover and six through mutations.

- In the Evo-devo $L$ the leg length $L$ grows in three developmental stages of each robot, while all other parameters are kept constant through development. The evolutionary process can, however, change the initial leg length of the robots through mutation and crossover. After the last stage of development, the fitness value for the "adult" robot is recorded. Thus, the leg length of the finally evaluated robot differs from the encoded value in its genotype ("child"), as it was grown in the developmental process. 
Each of the five generations consists of five agents that undergo three development stages, with a total of 15 fitnesses analysed per generation.

- Evo-devo LT is similar to the previous Evo-Devo case, but leg thickness is included as a Development parameter, and consequently excluded from the parameters to be modified during Evolution. Here, leg thickness always starts at the minimum value and grows constant thickness increments over the life span (Development), as shown in Eq. (4). The idea behind this developmental criterion is to mimic the ontogenetic growth from biological forms.

A comparison between Evolution and Evo-devo cases is not the focus of this paper, as the developmental search happens over a smaller search space, which would render such comparison unfair. Additionally, the idea behind development requires the emulation of ever-changing morphologies, where the "infant" morphology is selected based on the "adult" behavior, and such a process is deemed to produce different results from those observed in the Evolution case.

Both Evo-devo cases use the same initial seed, and these five initial genomes were randomly taken from the initial pool adopted at the evolution-only case. The high stochasticity from the contact between the robot and the environment yielded different fitnesses for similar cases.

Finally, we compare the gains associated with the developmental increments by creating a metric called $\delta f$, which compares the difference between first/second and second/third fitnesses from developmental stages (henceforth called as 1st and 2nd developments). The results of these comparisons are needed to assess in which conditions the developmental stage is improving the performance within Evo-devo cases. The equation is as follows:

$$
\delta f=f^{n+1}-f^{n}
$$

where $n$ represents the developmental stage.

The results for all developmental stages and generations were compared with a twoway ANOVA test considering a 95\% confidence interval, and a Tukey HSD was chosen as a post-hoc analysis to avoid a multiple comparisons error.

\section{Results and discussion}

\subsection{Evolutionary experiments}

In Fig. 5 the evolved robots are shown and the diversity between legged morphologies can be seen. The Evolution case explored a host of different leg designs and provided varied adaptations to reach a higher fitness function. Although limited by real-world constraints, a relatively wide range of leg angles, lengths, symmetries and walking gaits have been explored during this experiment.

The results of this experiment are shown in Fig. 6, with a chronological comparison of generations advancing horizontally. The data shows that the highest fitness value 
Vujovic et al.

Evolutionary Developmental Robotics

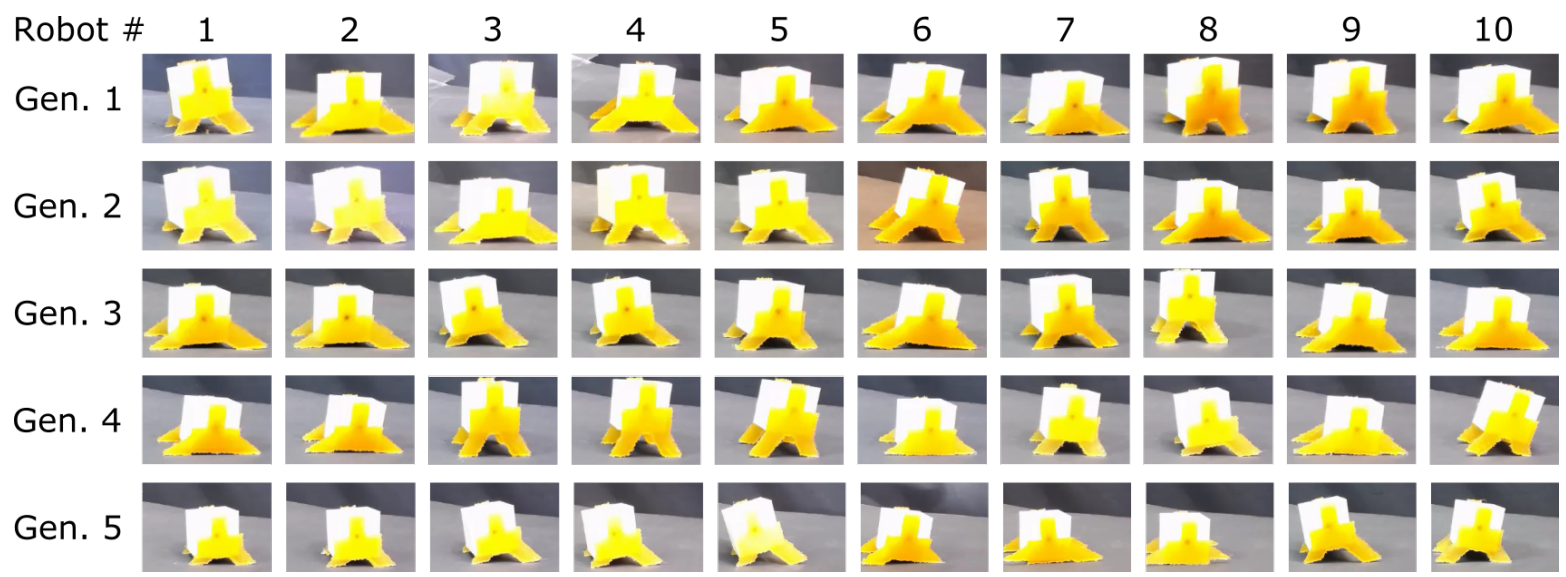

Figure 5. The legged robots evolved in the first evolutionary experiment exhibit a large variety of leg morphologies.

observed in this experiment is reached in generation two, where the legged robot walked almost $40 \mathrm{~cm}$, and the remaining three generations do not yield higher displacements. During this evolutionary algorithm the genetic crossovers and mutations explored variations of the best results (blue and red lines) to reach faster robots.

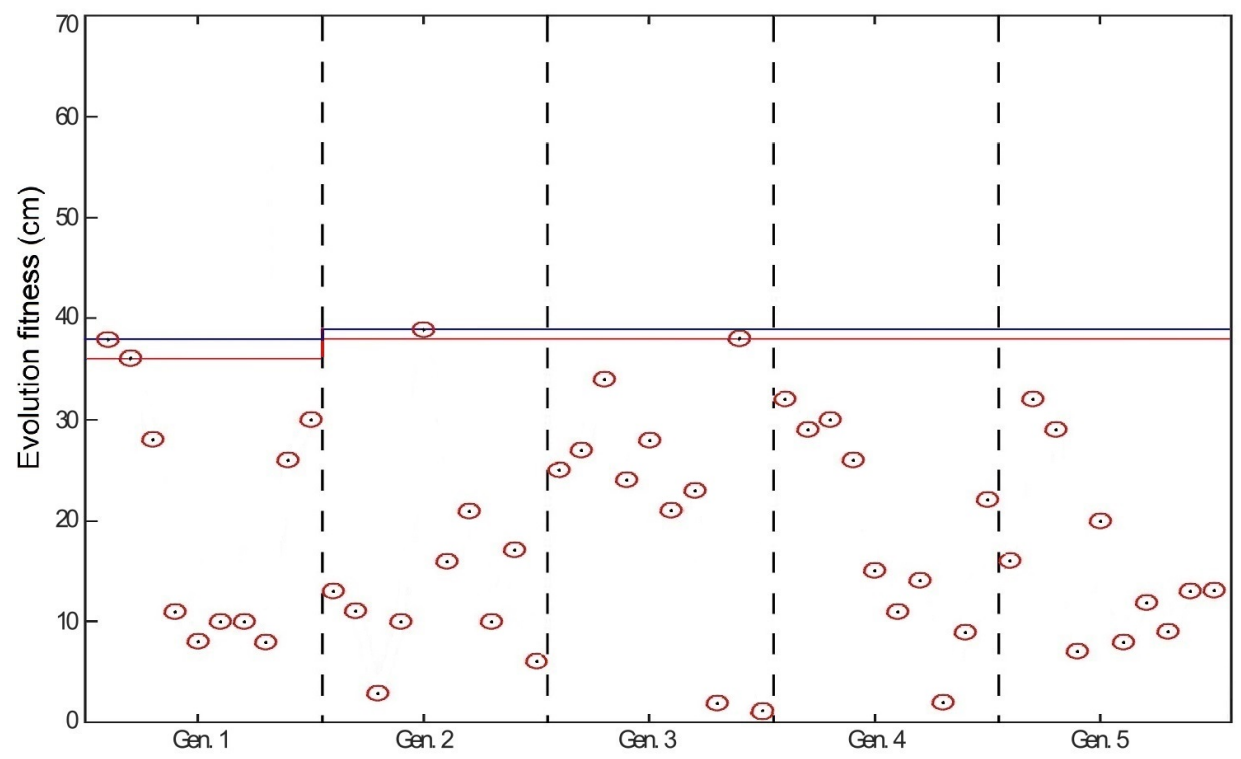

Figure 6. Evolution of robots over 5 generations. Each circle represents a single robot, their fitness values and their generation groups. The blue line on the top shows the maximum fitness achieved up to that generation and the red line the second best value. Thus, these lines indicate the individuals selected to form the offspring in the subsequent generation. Notably, the fitness function does not exceed $40 \mathrm{~cm}$.

The complexity of the search space, characterized by the non-linear relationship between length, angle and thickness, combined with the fitness evaluation in real-world experiments presumably caused the relatively low maximum fitness of this evolutionary experiment. 


\subsection{Evo-Devo experiments}

The influences of developmental processes were tested with two additional experiments. Within these experiments we evaluated five generations with five agents each (two generated via crossover and three through mutation), and the results from the two elite agents from previous generation were carried over for further reproduction. Both Evodevo cases adopt the same initial genetic seed and all robots from these experiments go through three developmental stages, which result in a total of 75 evaluations per experiment. The best adult fitness is chosen, in contrast to the best lifelong fitness, to mimic the partner selection process which only takes place at reproductive age.

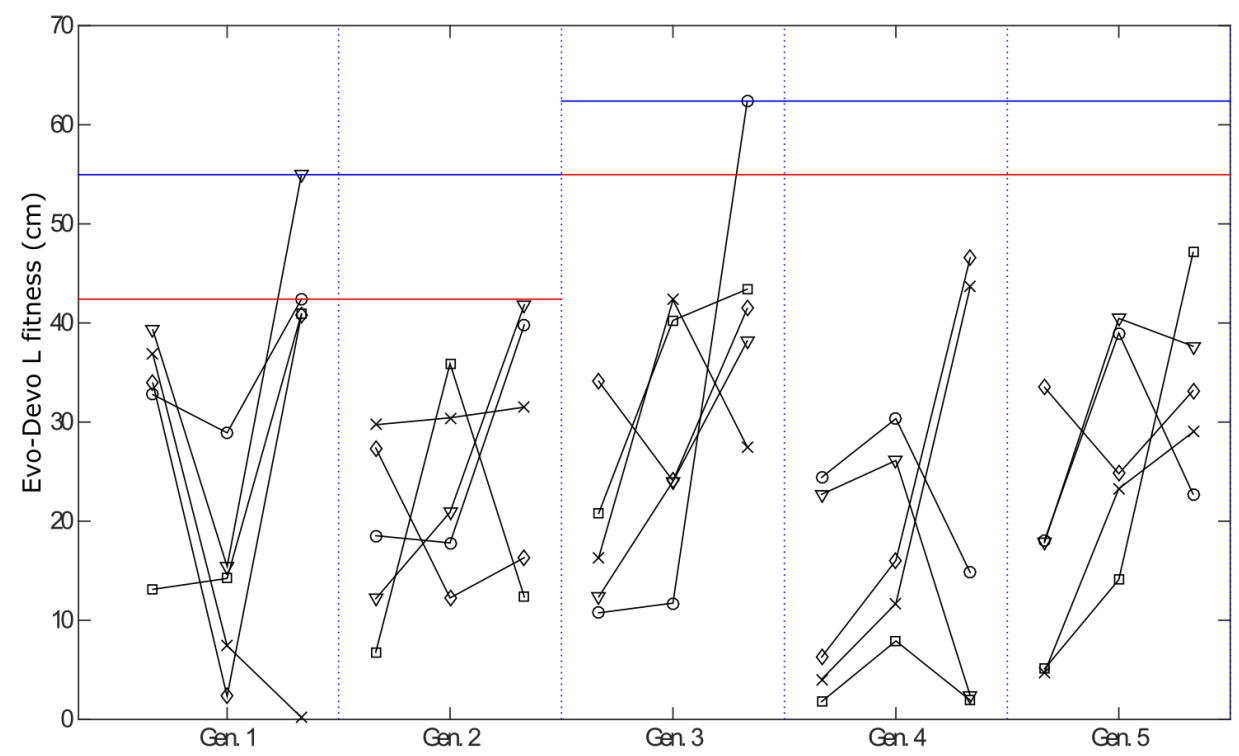

Figure 7. Evo-devo of robots over five generations, with development of leg length only. Within each generation, the results of all three developmental stages are shown, but only the last stage is considered for the selection. The blue line indicates the fittest individual up to the current generation, the red line the second best. Although every case started with a fitness value inferior to $40 \mathrm{~cm}$ (infancy) the developmental process allows the system to reach higher fitnesses (adulthood).

3.2.1. Developing leg length The results of this Evo-devo L experiment are shown in Fig. 7. There, the fitnesses of all three developmental stages for all evaluated robots are shown. The results show that the fittest robot was found in generation three, but developmental processes throughout all five generations improved fitnesses after growth. It can be seen in Tab. 2 that the majority of these results leads to an improvement of the robot's fitness, and especially at the third generation.

Overall, the Evo-devo L experiment presented solutions which improve with body growth, as fitnesses improved with the 1st and 2nd developments (Table 2). As the morphological growth is not embedded in the evolution, the evolutionary part is indirectly forced to choose infant states which will yield better adults. The nested developmental 
process allowed an internal parameter search (leg length) while freezing other parameters to achieve faster locomotion (higher absolute fitness).

Table 2. Success rate of L Developmental stages. A successful growth is characterized by the association between increases in fitness value and morphology.

\begin{tabular}{|l|c|c|c|c|c|}
\hline \hline Growth type & Gen 1 & Gen 2 & Gen 3 & Gen 4 & Gen 5 \\
\hline \hline Stage to stage & $5 / 10$ & $7 / 10$ & $8 / 10$ & $7 / 10$ & $7 / 10$ \\
Start to end of development & $4 / 5$ & $4 / 5$ & $5 / 5$ & $3 / 5$ & $4 / 5$ \\
\hline \hline
\end{tabular}

3.2.2. Developing leg length and thickness The results for the Evo-devo LT case demonstrated the impact of both leg length and thickness on the fitness evaluation, as seen in Fig. 8. Unlike the leg length, which was grown depending on the fitness from an initial starting value (encoded in the robot's genome), the leg thickness was no longer under evolutionary control, as it always started with two layers at the first developmental stage and grew to four layers at the third stage for all robots.

The experiment was conducted with the same parameters from the Evo-devo L experiment, thus 75 legged robots were evaluated in total $(5$ generations $\mathrm{x} 5$ robots $\mathrm{x}$

Gen. 1

Robot 1

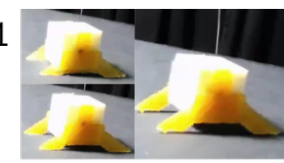

Gen. 2

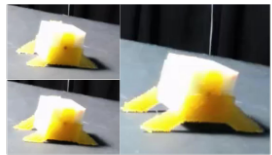

Gen. 3

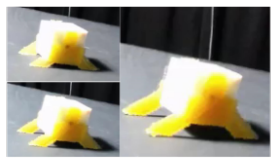

Gen. 4

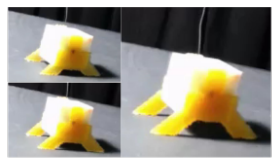

Gen. 5

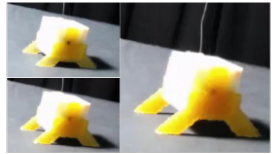

Developmental stages
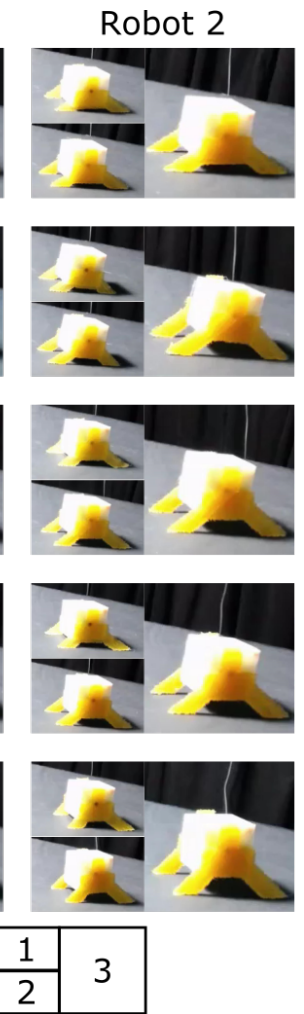

3
Robot 3
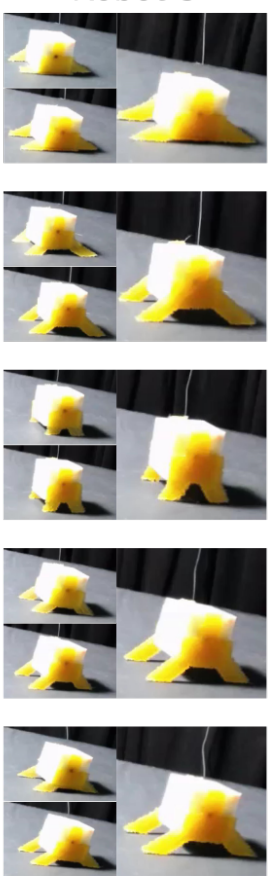

Robot 4
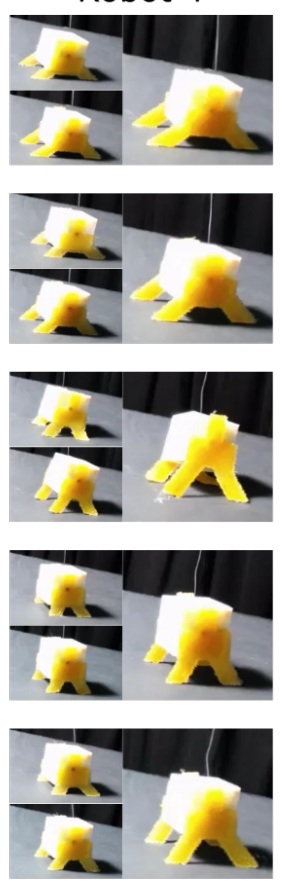

Robot 5
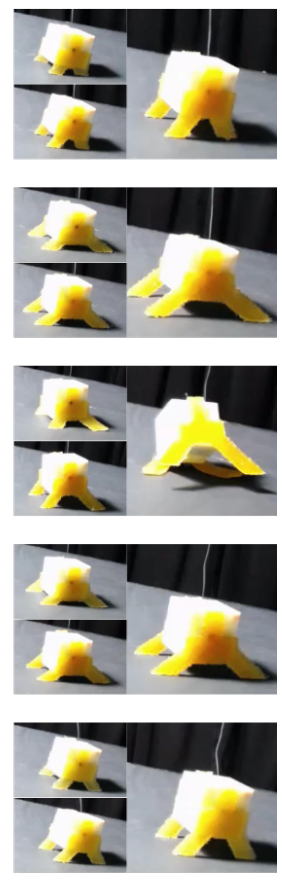

Figure 8. Legged robots evolved with development of leg length and thickness. All three developmental stages of the evolved robots are shown. The robots grow from the 1 st to the $2 \mathrm{nd}$, and lastly to the 3rd stage. 


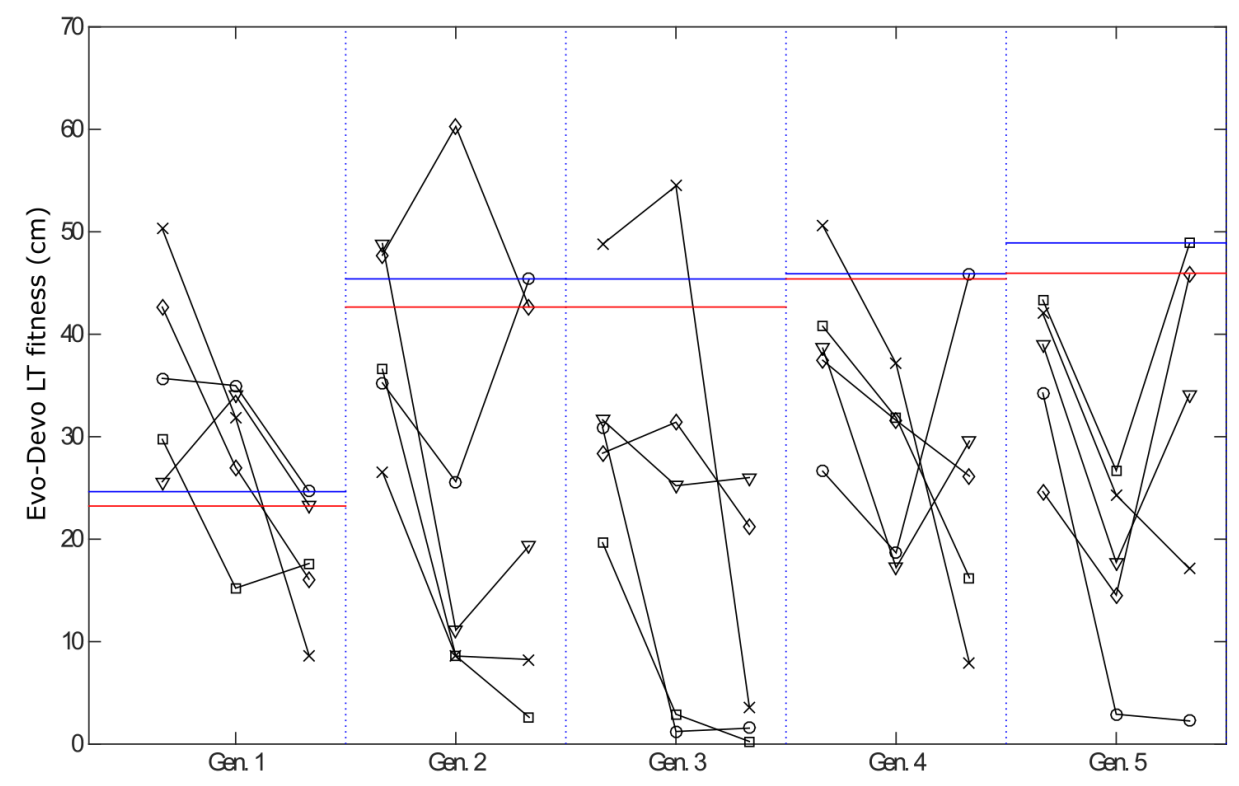

Figure 9. Evo-devo of robots over five generations, with the development of leg length and leg thickness. The results of all three developmental stages are shown within each generation, but only the last stage is considered for the fitness selection. The blue line indicates the fittest individual up to the current generation, the red line the second best. Many "adult" individuals perform poorer than their younger states.

3 developmental stages). Fig. 9 shows the results of this experiment, with generations of individuals evolving and developing with time. The analysis of these results shows that this choice of parameters on the developmental process has a detrimental effect on the final fitness, where the vast majority of individuals perform better during their early age (infancy) than at the latest stages of growth (adulthood), as shown in Tab. 3.

Table 3. Success rate of LT developmental stages

\begin{tabular}{|l|c|c|c|c|c|}
\hline \hline Growth type & Gen 1 & Gen 2 & Gen 3 & Gen 4 & Gen 5 \\
\hline \hline Stage to stage & $2 / 10$ & $3 / 10$ & $1 / 10$ & $2 / 10$ & $3 / 10$ \\
Start to end of development & $0 / 5$ & $1 / 5$ & $0 / 5$ & $1 / 5$ & $2 / 5$ \\
\hline \hline
\end{tabular}

\subsubsection{Comparison between developments Comparing the developmental alternatives} for the Evo-devo cases, a statistical analysis shows that the Evo-devo L case is superior to the Evo-devo LT case $(\mathrm{p}<0.0001)$ and, overall, the second developmental stage is superior to the first stage (statistically significant with $\mathrm{p}=0.0137$ ), as seen in Fig. 10 . Moreover, while the Evo-devo L case had a clear tendency of improving the fitness value with development stages, the Evo-devo LT demonstrated a strong tendency in degrading the performance $(\delta f<0)$ with growth. 


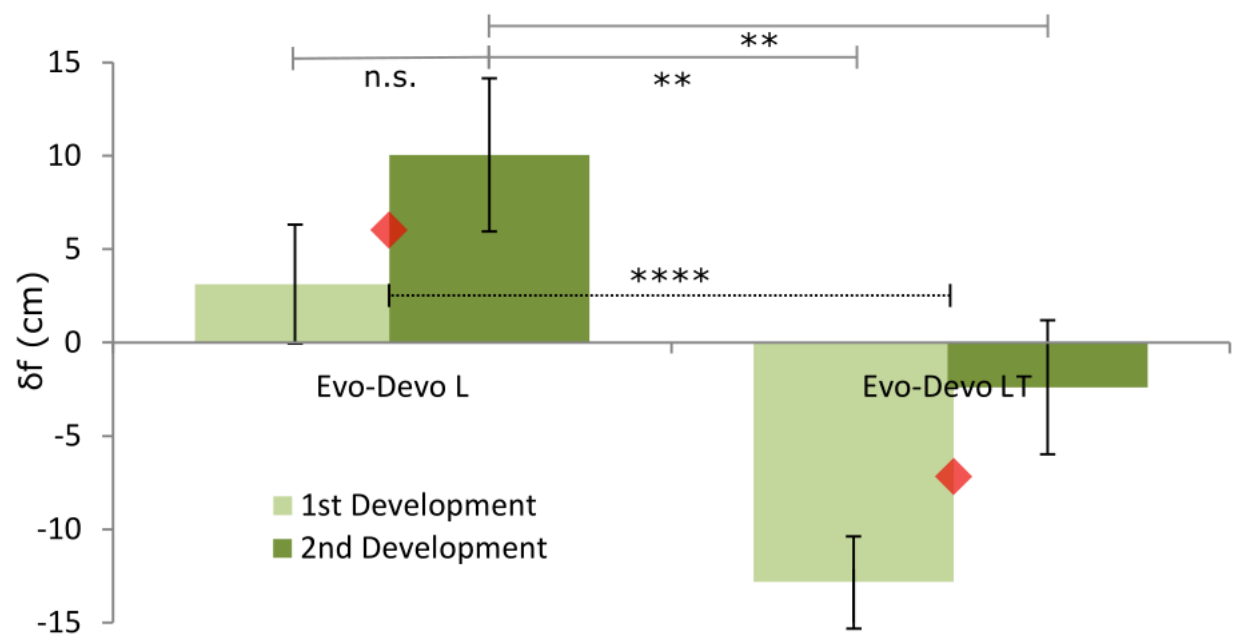

Figure 10. This figure depicts the means for both Evo-devo cases. The red rhombus shows the overall average fitness of each case, and **** represents $p<0.0001$, ** represents $p<0.01$ and n.s. represents that no statistical significance was obtained. The case Evo-Devo L outperformed the case Evo-Devo LT, and the developmental stages of the case Evo-Devo L resulted in positive fitness increments on average and contrasted to the case Evo-Devo LT, as this one resulted in negative fitness increments on average.

3.2.4. Locomotion behavior Throughout the above experiments, a range of different leg morphologies were evolved. Based on these diverse morphologies, a large range of locomotion behaviors emerged during the fitness evaluation, and several distinct behaviors can be identified from the trajectories, as shown in Fig. 12. As one might expect, both straight and curved trajectories were observed, but also more complex behaviors such as S-shaped trajectories or U-turns. Moreover, we could observe locomotion in two different directions: forward and sideways (with respect to the active module's orientation).

The elasticity introduced by the glue legs might have caused such variability, and such diversified behavior fortify our claims about the difficulty in simulating such results within a computer environment. The use of real-world data to create new robots is a strong trend for the future, either by the invention of robots capable of changing their own morphology and adapting their control to it, or by a real-world design optimization method to guarantee better robots as final output.

\subsection{Discussion}

The results of these three experiments demonstrated the possibility of creating of legged robots through evolution and, for the first time in robotics, through an Evo-devo process which contains a nested developmental search. These results show that the Evo-devo system is capable of finding solutions to improve the locomotion of growing robots, which is in agreement with the seminal review from Lungarella et al. over development 

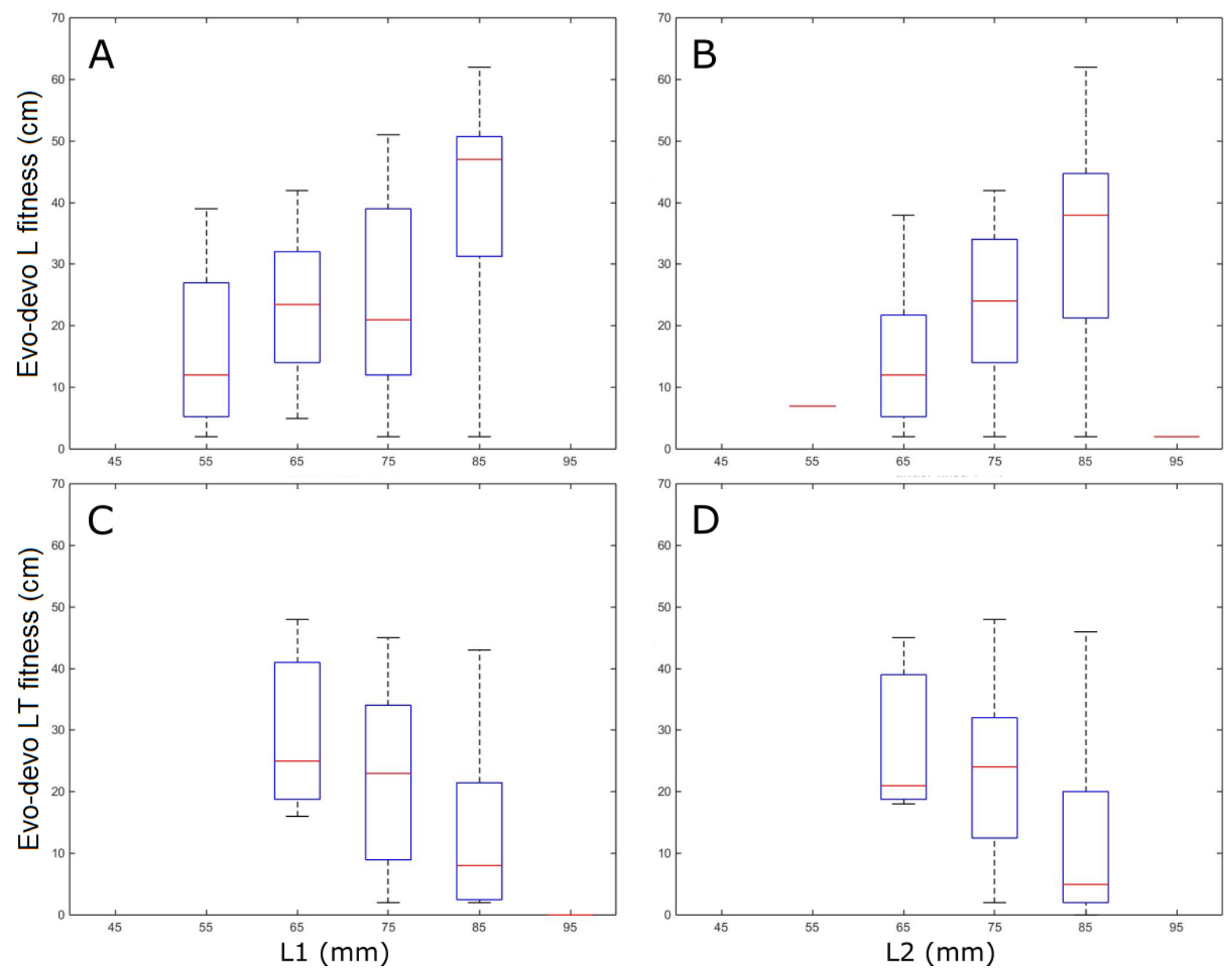

Figure 11. Fitness values for different leg lengths $L 1(\mathrm{~A}, \mathrm{C})$ and $L 2(\mathrm{~B}, \mathrm{D})$ with fixed thickness $T=4$. The plots in the top row $(\mathrm{A}, \mathrm{B})$ show the results for leg length development only. The second row $(\mathrm{C}, \mathrm{D})$ shows the results for the combined development of leg length and thickness. The observed trends were not true for thinner legs $(T \leq 3)$

in simulated environments [18]. A comparison between the two Evo-devo experiments provides interesting questions for discussion.

Comparison between Evo-devo cases As Fig. 10 shows, the increase in fitness values due to development is larger in the Evo-devo L case than in the Evo-devo LT experiment. A lower maximum fitness was also observed in the latter, where leg length and thickness were grown during the development. This finding is surprising, as the developmental search was added to freeze certain angle and control parameters and further increase the fitness. The question that arises is how leg length $L$ and thickness $T$ are coupled and how they relate to the locomotion fitness. In Blickhan [23] it is suggested that leg morphologies during locomotion can be simplified as springs, and in this aspect we could infer that thicker legs would represent stiffer springs. Although intuitively we would accept the idea that longer legs invariably produce faster strides, often normalized 

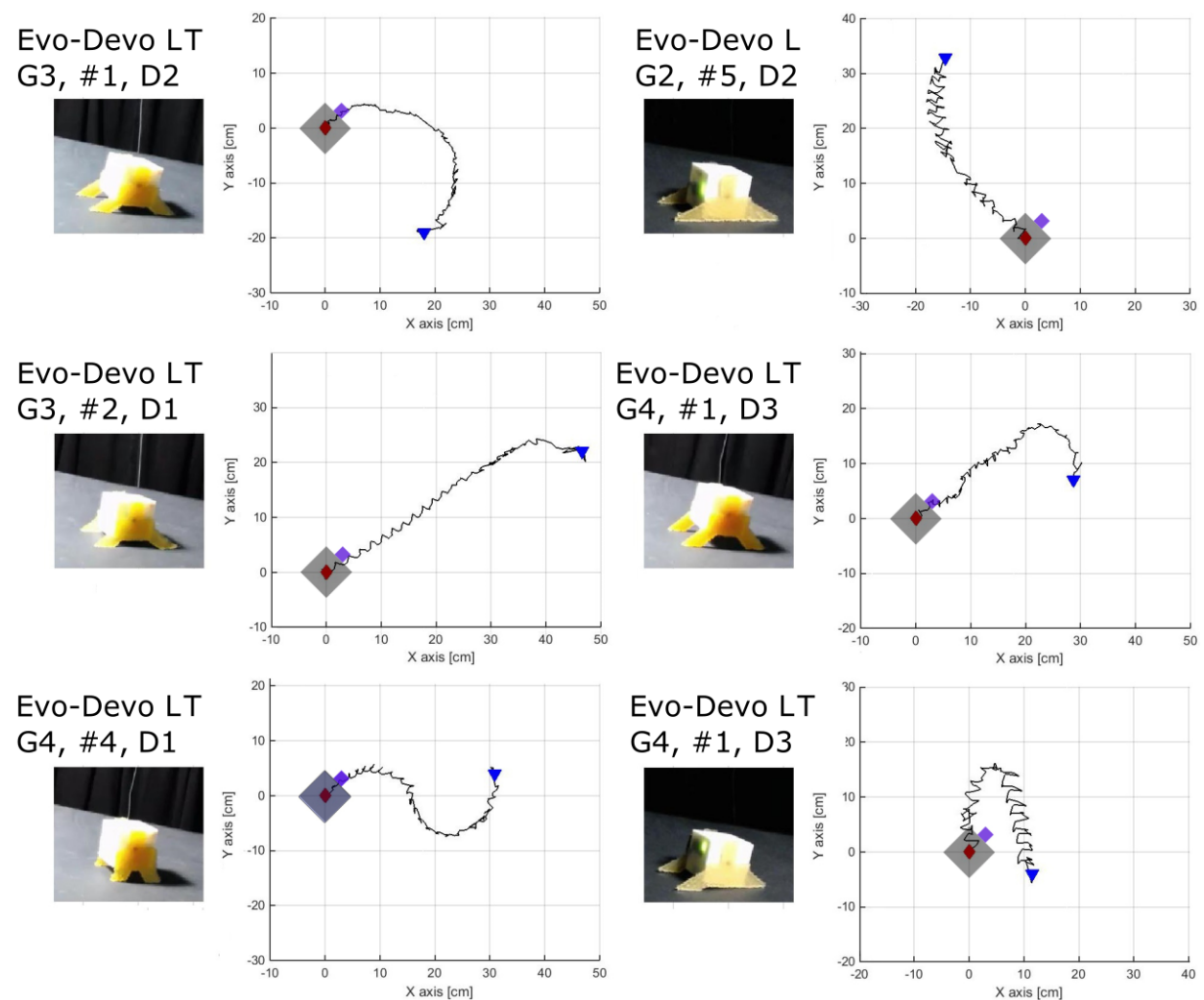

Figure 12. The above plots show the trajectories of six evolved legged robots which were extracted from the overhead camera footage of the fitness evaluation. The trajectories reveal a broad range of locomotion behaviors such as curving front or sideways (first row), straight locomotion and straight locomotion with curve at the end (middle row) and S-shape curve and U-turn trajectory (bottom row). Each individual is depicted by their Evo-devo case, generation, number and developmental stage.

by the Froude number for size comparisons [24], the delicate interplay between length and thickness from this work would indicate a complex relationship, as demonstrated in Fig. 11. There, the right and left leg lengths $L_{1}$ and $L_{2}$ similarly showed that Evo-devo LT and Evo-devo L have opposing behaviors: A positive correlation between leg length and fitness for one is countered by a negative correlation for the other.

During the Evo-devo LT case the developmental process forcefully leads to an increase of both leg length and thickness from one stage to another. We hypothesize that long and thick legs do not reap the locomotive benefits described in [23], and this explains the negative influence of a forceful increase of thickness during the developmental process, corroborated by Fig. 10.

Developmental parameter selection While the benefits from Evo-devo were discussed previously, the influence of the developmental stage can be damaging if not properly set: The Evo-devo LT cases were marked by detrimental growth changes (Fig. 10), and we hypothesize that the search space reduction eventually removed the "good choices" that development could have chosen by enforcing an incompatible thickness $T$ for each 
stage. In the past, Bongard has found a similar influence within computer simulations [9], and our results also show that "the right kind of morphological change accelerates the discovery of successful behaviors."

An analysis of walking animals [23] hints to a correlation between length and thickness, which is what Evo-devo LT cases adopted. Thicker legs have higher natural frequencies, while longer legs have lower frequencies, and a combined growth of these two might create a longer and thicker leg with a similar natural frequency. Although length and thickness were not proportionally coupled, the lower performance of Evo-devo LT cases when compared to Evo-devo L cases leads us to believe that higher fitnesses are not associated with one specific natural frequency.

The fitness values during the Evolution case might have been hindered by the presence of too many parameters and thus the genotype-to-fitness mapping becoming overly complex. Koos et al. address a similar problem whilst closing the reality gap [26], as the correlation between such parameters can be highly non-linear, and Jakobi et al. emphasize the importance for such parameters to be wisely chosen [19]. Therefore, it remains open for discussion how a suitable growth function can be designed, and how to assign design parameters to the evolutionary or developmental processes. Our aim with this work was to offer a new perspective to adaptive robotics by introducing Evo-devo processes with a higher emphasis on the devo aspect of it, which is fairly unexplored in real-world environments. Despite our efforts, much still has to be done to understand how both evo and devo parameters affect the final performance.

Evolution and Evo-devo considerations A direct comparison between Evolution and Evo-devo cases is counter-intuitive, as Evo-devo cases try to find the child which will become the best adult, while evolution is solely focused on the best individual overall . However, both developmental approaches surpassed the maximum fitness value of "40 cm" within the first generations, and although not specifically searching for the best individual it presented itself as useful to force the system into exploring specific traits associated with growth while keeping the other traits untouched, as suggested in [9]. Our nested developmental approach considered a local search over a set of parameters, and there might be a resemblance between this and memetic algorithms when the local improvement aspects are considered. Evolution cases were not forced into a growth pattern, and the search space remained open for the entirety of the experiment, while the Evo-devo cases "froze" part of their search space during growth stages.

Within the Evo-devo experiments the best genome is chosen by choosing the best adult fitness instead of the best lifelong fitness. While our intention with this choice is to mimic the partner selection process in a sexual evolution, which only takes place at reproductive age, the effects of this choice will be further investigated in future experiments.

Our experiments solely considered the learning of the robot which was creating smaller legged robots, not from the legged robot itself, and our next works will approach the possibility of legged robots adapting their control method to maximize their 
movement. Another future consideration will be on the consequences of the hereditary transfer of optimized control genes in these robots (i.e. Lamarckian evolution).

\section{Conclusion}

Motivated by the way plants and animals adapted to their environments through evolution and by their adjustment to external influences on an ontogenetic timescale, this article presents results from the first robot in the world which combines evolutionary and developmental processes. It can autonomously design, build, test and improve the morphology and control of legged robots. In order to avoid the pitfalls of simulation, all solutions are physically tested during their evaluation, as many researchers from the same field struggle in addressing such Reality Gap [19, 26]. To enable a large variety of shapes, the legged robots are not only assembled from a set of predefined parts, but can also be additively fabricated in real time with different lengths and stiffnesses.

The comparison between an evolutionary design and the Evo-devo strategy, which combines a developmental search with the evolutionary design of locomotion agents, should be formally addressed in the future. However, our experiments indicate that the addition of an internal development search (Evo-devo) resulted in "adult" legged robots with a higher fitness by freezing certain design parameters. Additionally, a comparison between the two Evo-devo experiments with different developmental parameters reveal the importance of choosing the right parameters to evolve and to develop.

The interplay between development and evolution during this process is complex and not yet fully understood. However, the desired improvement in fitness can only emerge if the evolutionary and developmental terms are properly adjusted, as seen within our experiments. Amongst the important design choices are the selection of developmental parameters and their respective growth functions. Here, two parameters were considered - leg length and leg thickness - each with a specific growth function. Leg length developed depending on the fitness of the agent in the preceding stage. Fit agents achieved little growth (only minor changes to well working robots), while slow robots achieved a higher growth of leg length. On the other hand, the leg thickness was increased according to a fixed plan, adding one layer from one stage to the next.

The development of the leg length achieved the best results, while the combination with the development of leg length and leg thickness did not render strong "adult" robots. However, more data is required to assess whether this drop in performance is induced by too many parameters within development, where the genotype-tofitness mapping would be too complex, or whether a different choice of developmental function could have improved performance. Thus, it remains as future work to derive the appropriate design rules and guidelines, such that the Evo-devo process can be successfully applied to more complex robot design problems. 


\section{Acknowledgements}

This research was supported by the RoboSoft - Coordination Action for Soft Robotics, funded by the European Commission under the Future and Emerging Technologies (FP7-ICT-2013-C project no 619319).

\section{References}

[1] A. Cully, J. Clune, D. Tarapore, and J.B. Mouret Robots that can adapt like animals. Nature, 521(7553):503-507, 2015.

[2] A. P. Moczek, S. Sultan, S. Foster, C. Ledón-Rettig, I. Dworkin, H. F. Nijhout, E. Abouheif, and D. W. Pfennig. The role of developmental plasticity in evolutionary innovation. Proc. R. Soc. $B, 278(1719): 2705-2713,2011$.

[3] D. Floreano and F. Mondada Evolution of homing navigation in a real mobile robot. IEEE Trans. on Systems, Man and Cybernetics, 26(3):396-407, Jun. 1996.

[4] G Hornby, J Lohn, and D Linden. Computer-automated evolution of an x-band antenna for NASA's space technology 5 mission. Evolutionary Computation, 19(1):1-23, 2011.

[5] H. E. Hoekstra and J. A. Coyne. The locus of evolution: Evo devo and the genetics of adaptation. Evolution, 61(5):995-1016, 2007.

[6] J. Hiller and H. Lipson. Automatic design and manufacture of soft robots. IEEE Trans. Robot., 28(2):457-466, Apr. 2012.

[7] J.C. Bongard, V. Zykov, and H. Lipson. Resilient machines through continuous self-modeling. Science, 314(5802):1118-1121, 2006.

[8] J.C. Bongard. Evolutionary robotics. 56(8):74-83, August 2013.

[9] J.C. Bongard. Morphological change in machines accelerates the evolution of robust behavior. Proc. Natl. Acad. Sci., 108(4):1234-1239, 2011.

[10] J. Clune, B.E. Beckmann, C. Ofria, and R.T. Pennock. Evolving coordinated quadruped gaits with the hyperneat generative encoding. In Evolutionary Computation, 2009. CEC 'O9. IEEE Congress on, pages 2764-2771, May 2009.

[11] J.D. Hiller and H. Lipson. Evolving amorphous robots. In Proceedings of the Twelfth International Conference on Artificial Life (ALIFE XII), 2010.

[12] J. Rieffel, D. Knox, S. Smith, and B. Trimmer. Growing and evolving soft robots. Artificial Life, 20(1):143-162, February 2014.

[13] J. M. Guayasamin, T. Krynak, K. Krynak, J. Culebras, and C. R. Hutter. Phenotypic plasticity raises questions for taxonomically important traits: a remarkable new andean rainfrog (pristimantis) with the ability to change skin texture. Zoological Journal of the Linnean Society, 173(4):913-928, 2015.

[14] K. Sims. Evolving 3D morphology and behavior by competition. Artificial Life, 1(4):353-372, 1994.

[15] L. Brodbeck, S. Hauser, and F. Iida. Morphological evolution of physical robots through model-free phenotype development. PLoS ONE, 10(6):e0128444, 062015.

[16] L. Wang, L. Brodbeck, and F. Iida. Mechanics and energetics in tool manufacture and use: a synthetic approach. J. R. Soc. Interface, 11(100), 2014.

[17] M. Joachimczak, R. Suzuki, and T. Arita. Fine grained artificial development for body-controller coevolution of soft-bodied animats. Proc. of the Intl. Conf. on Synthesis and Simulation of Living Systems, 239-246, 2014.

[18] M. Lungarella, G. Metta, R. Pfeifer, and G. Sandini. Developmental robotics: a survey. Connection Science, 15(4):151-190, 2003.

[19] N. Jakobi, P. Husbands, and I. Harvey. Noise and the reality gap: The use of simulation in evolutionary robotics. In Federico Morán, Alvaro Moreno, Juan Julián Merelo, and Pablo 
Chacón, editors, Advances in Artificial Life, volume 929 of Lecture Notes in Computer Science, pages 704-720. Springer Berlin Heidelberg, 1995.

[20] R. Gross, M. Bonani, F. Mondada, and M. Dorigo. Autonomous self-assembly in swarm-bots. IEEE Trans. Robot., 22(6):1115 -1130, dec. 2006.

[21] J. Rieffel, and D. Sayles. EvoFab: A Fully Embodied Evolutionary Fabricator. In Gianluca Tempesti, Andy M. Tyrrell, and Julian F. Miller, editors, Evolvable Systems: From Biology to Hardware, volume 6274 of Lecture Notes in Computer Science, pages 372-380. Springer Berlin Heidelberg, 2010.

[22] R. Doursat and C. Sánchez. Growing fine-grained multicellular robots. Soft Robotics, 1(2):110121, June 2014.

[23] R. Blickhan, A. Seyfarth, H. Geyer, S. Grimmer, H. Wagner, and M. Gunther. Intelligence by mechanics. Phil. Trans. R. Soc. A, (365):199-220, 2007.

[24] R. McN. Alexander. Principles of Animal Locomotion. Princeton University Press, Princeton, 2013.

[25] S. Nolfi and D. Floreano. Evolutionary robotics: The biology, intelligence, and technology of selforganizing machines. MIT Press, Cambridge, MA, 2000.

[26] S. Koos, J.-B. Mouret, and S. Doncieux. The transferability approach: Crossing the reality gap in evolutionary robotics. IEEE Trans. Evol. Comput., 17(1):122-145, 2013.

[27] S. B. Carroll. Evo-devo and an expanding evolutionary synthesis: A genetic theory of morphological evolution. Cell, 134(1):25 - 36, 2008.

[28] S. E Sultan. Phenotypic plasticity for plant development, function and life history. Trends in Plant Science, 5(12):537-542, 2000.

[29] S. S. Crump. Fused deposition modeling (FDM): putting rapid back into prototyping. In The Second International Conference on Rapid Prototyping, pages 354-357, 1991.

[30] Y. Jin and Y. Meng. Morphogenetic robotics: An emerging new field in developmental robotics. IEEE Trans. Syst., Man, Cybern. A, 41(2):145-160, March 2011. 